Voix et Images

voixetimages

\title{
Pourquoi se raconter?
}

\section{Robert Major}

Volume 24, numéro 2 (71), hiver 1999

Poésie québécoise et histoire littéraire

URI : https://id.erudit.org/iderudit/201435ar

DOI : https://doi.org/10.7202/201435ar

Aller au sommaire du numéro

\section{Éditeur(s)}

Université du Québec à Montréal

\section{ISSN}

0318-9201 (imprimé)

1705-933X (numérique)

Découvrir la revue

\section{Citer cet article}

Major, R. (1999). Pourquoi se raconter? Voix et Images, 24(2), 399-406. https://doi.org/10.7202/201435ar d'utilisation que vous pouvez consulter en ligne.

https://apropos.erudit.org/fr/usagers/politique-dutilisation/ 


\title{
Essai
}

\section{Pourquoi se raconter?}

\author{
Robert Major, Université d'Ottawa
}

"Cher fils...": ainsi commence, en 1771 , la célèbre autobiographie de Benjamin Franklin, conçue comme une longue épître à son fils aîné William. Ayant lui-même toujours été très curieux de ses origines et soucieux d'obtenir le plus de renseignements possible sur ses ancêtres, Franklin suppose que son fils sera intéressé par le récit qu'il entame. Et d'autant plus que la trajectoire de sa vie, d'une pauvreté anonyme à la célébrité comme écrivain, scientifique et homme d'état, confère à son destin une certaine valeur exemplaire: son fils pourra y glaner des leçons de vie tout en satisfaisant la curiosité naturelle que nous avons tous sur notre lignage.

Benjamin Franklin ne pouvait se douter qu'à peine quatre ans plus tard, père et fils seraient dans des camps ennemis, le premier choisissant les États-Unis d'Amérique, dont il allait devenir père fondateur, le second optant pour le loyalisme et la vie en Angleterre. Qu'à cela ne tienne: Franklin a continué d'écrire, père d'une République mais orphelin de fils, ayant perdu son destinataire initial et privilégié; ayant perdu, du même coup, le prétexte narratif qu'il avait utilisé. Par ailleurs, il n'avait pas perdu sa vanité, qu'il reconnaissait d'emblée dès la première page de son récit; ni le goût qu'ont toutes les personnes d'un certain âge de se remémorer les souvenirs d'une longue vie; ni, sans doute, la conscience obscure qu'il devait raconter pour la postérité, sous peine de laisser dans l'ombre quelque chose d'essentiel.

Franklin n'est pas de ces êtres "de qui personne ne parlera si euxmêmes n'en parlent ", comme disait Montaigne pour justifier le contenu autobiographique de ses Essais. On aurait parlé de lui néanmoins, tant il domine son époque. Et il aura réussi son autobiographie comme le reste. L'image de Franklin m'est revenue en mémoire en lisant le très beau livre de Fernand Dumont, Récit d'une émigration. Mémoires ${ }^{1}$. Tout distingue les deux êtres, il me semble, du moins au niveau du caractère et du persona public, et pourtant les ressemblances et les dissemblances de leurs trajectoires les rapprochent et éclairent quelque peu ce livre ultime de l'un de nos grands intellectuels. Le même passage des classes laborieuses à la culture savante, la même curiosité pour la raison ultime des choses, le même sens aigu de l'engagement social, le même sentiment de participer à l'avènement d'une nouvelle société - avènement réussi dans le cas de l'Américain, toujours reporté dans le cas du Québécois -, la même 
conviction intime d'être, pour l'essentiel, à travers toutes les personnes rencontrées et les livres lus, malgré tout un autodidacte, et donc de livrer le récit d'une éducation. Nous sommes peut-être devant une forme typiquement américaine de l'autobiographie: "mémoires d'une vie éduquée", où l'auteur, tout en se composant à travers le récit de sa vie, comme c'est le cas pour toute autobiographie, détaille également le passage de l'état de nature (ou ce qui en tient lieu, la culture populaire séculaire) à l'état de culture. Dans un contexte de démocratie où le mérite peut se faire reconnaitre et assure seul le succès. Fernand Dumont, dont la vie studieuse et réflexive s'est passée dans l'enceinte de l'université, serait peut-être très étonné de se voir rapproché de ce Yankee affairiste, remuant et ambitieux. Et peut-être pas, non plus.

Comment parler de son livre important mais qui concerne, dans un premier temps du moins - et si on exclut les intimes, les collègues immédiats et les collaborateurs surtout les sociologues, les anthropologues, les théoriciens de la culture, à la limite les historiens de la religion et les théologiens? Comme un littéraire, attentif au statut littéraire du texte, à sa qualité d'écriture, à l'universalité de son propos, débordant les cloisons des disciplines et les frontières savantes.

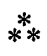

Le genre de l'autobiographie est certainement la province la plus démocratique de la république des lettres, puisque politiques et littéraires, illettrés et savants, adolescents et vieillards, criminels et héros, et à la limite tout un chacun peut choisir de raconter sa vie à son magnétophone pour ensuite transcrire le récit ou le faire transcrire. Il est heureux qu'il en soit ainsi, d'ailleurs. Même les êtres les plus simples et les plus apparemment ordinaires recèlent quelques récits qui importent: ce sont les récits de vie que traquent maintenant anthropologues, sociologues, cinéastes et romanciers. Le roman occidental qui, depuis plus d'un siècle, dans la foulée du réalisme, a délaissé les héros exceptionnels pour s'intéresser aux obscurs quidams, qui avait commencé à le faire bien avant, d'ailleurs, en pratiquant le roman picaresque, n'a pas d'autre credo: tout récit intéresse, s'il est bien raconté; toute vie humaine contient des mystères qui sont autant de filons pour les écrivains. D'où notre intérêt pour le récit de vie. À plus forte raison si la personne qui raconte a une valeur exemplaire ou incarne dans son destin une dimension importante de celui de la collectivité ou encore peut formuler ce qui autrement resterait ineffable ou diffus. C'est le cas avec Fernand Dumont.

Mais sans doute faut-il lever au départ une hypothèque. L'insistance sur le récit, dans mes propos introductifs comme dans le titre même du livre de Fernand Dumont, pose d'emblée la question de l'appartenance de l'autobiographie à l'espace de l'essai, et donc la pertinence de sa présence dans cette chronique. Question difficile, que je n'ai pas la prétention de résoudre, mais dont l'examen sommaire permettra peut-être d'éclairer les approches du livre de Fernand Dumont. L'autobiographie (je m'en 
tiens aux autobiographies réelles, car les fausses sont nombreuses) n'est pas un texte fictif. Du moins, elle prétend ne pas l'être. Mais on sait que, dans la mesure où l'auteur ne manipule que des données conscientes et n'offre que ce dont il veut parler, le dévoilement reste limité; le lecteur moderne, surtout frotté de psychanalyse, n'en est pas dupe. Dans ces circonstances, est-ce toujours un récit de la réalité?

Admettons, pour faire court et pour nous plier au sens commun, que l'autobiographie n'est pas fiction et se situe dans un autre registre que le roman. Elle partage néanmoins avec ce dernier de nombreux traits: le récit, donc l'utilisation de procédés narratifs, un héros, des personnages, des décors, une chronologie. D'ailleurs, de nombreux romans empruntent la structure fondamentale de l'autobiographie: un narrateur, qui est en même temps le héros de son récit. On dira que dans le cas de l'autobiographie, il y a conjonction du narrateur, de l'auteur et du héros, mais rien n'est moins sûr. L'autobiographie recompose du sens $a$ posteriori; le narrateur est coupé de son héros (cet autre lui-même) par le temps et le savoir; il a une vision d'ensemble de son destin; ou, du moins, il cherche à donner du sens aux événements qu'il a vécus, à l'époque, dans l'incertitude ou la confusion. L'autobiographie est un acte littéraire d'autoperformance dramatique par lequel un "Je" se construit dans le monde et façonne le passé pour en faire un présent qui a une certaine vérité symbolique. Ce serait alors naïf de croire à une fusion des trois entités auteur, narrateur, héros.
On le voit, les choses peuvent aisément se compliquer quand on examine l'autobiographie à l'aune du roman, mais là n'est pas mon propos. Partageant de nombreux traits avec le roman, l'autobiographie est-elle néanmoins un essai?

Certains jugeront que la question est sans intérêt ${ }^{2}$, sauf pour les spécialistes de l'essai, et que ces distinctions génériques ou ces querelles de frontières n'importent guère quand vient le moment de considérer l'œuvre vivante. C'est méconnaître la fécondité des genres, leur adéquation avec le mouvement fondamental de la création. Les genres ne sont pas que catégories d'école, conçues pour satisfaire la manie classificatoire des auteurs de manuels (ou des directeurs de revue). Ils répondent aux impératifs mêmes de l'écriture et en retour façonnent celle-ci de multiples façons.

Reconnaissons d'emblée que l'essai a des frontières poreuses et que l'essayistique, théorisation relativement récente d'un genre mouvant, a souvent fort à faire pour que cette étiquette ne devienne pas un fourretout commode de la prose d'idées, incluant pêle-mêle tout ce qui n'est pas "autre chose" de bien connu (roman, poésie, théâtre) ${ }^{3}$. Les cas d'hybridité générique sont nombreux: essai dans le roman et même dans la poésie, écriture poétique de l'essai, etc. Ceci dit, l'autobiographique et ses avatars - journal intime, correspondance, mémoires appartiennent bien, il me semble, du moins dans certaines de leurs incarnations exemplaires, à une forme spécifique d'essai. Dès sa création, l'essai a partie liée avec l'autobiographie. "Je suis moi-même 
la matière de mon livre", disait déjà Montaigne, ce qui portait Hugo Friedrich, dans son étude remarquable, à croire que Montaigne ne trouvait "digne de sa pensée qu'une idée chevillée aux fibres de son cœur $^{4}$ ". Tout lecteur de Montaigne est à même de constater que celui-ci s'aventure volontiers dans le récit et conçoit la vérité comme étant intimement liée à sa personne. La discussion d'idées, l'examen discursif d'une question dans les Essais est presque toujours lié à ses expériences, à ses états d'âme, à ses lectures. Montaigne, constamment, émaille ses propos de confidences, d'aventures vécues, de dialogues rapportés, de récits imagés. Ainsi, la pulsion autobiographique est aux sources mêmes de l'essai. Les meilleures autobiographies, d'ailleurs, partagent quelques-unes des préoccupations essentielles de l'essai : passer de l'apparence à une essence, d'une surface à une profondeur, découvrir, sous les hasards de la diachronie et la banalité de l'anecdotique, les structures typiques et le sens d'un parcours, trouver un style qui puisse rendre compte de cela.

L'autobiographie oscille, par définition, entre deux espaces opposés mais complémentaires: vers l'extérieur, l'expérience remémorée; d'une part; vers l'intériorité, la conscience réflexive, d'autre part. Ce mouvement de balancier confère à l'autobiographie sa tension génératrice, propre à l'essai. En somme, le véritable autobiographe - pour reprendre les catégories de Lukács quand il discute de l'essai - veut atteindre, par l'analyse d'une vie, la sienne, l'intériorité-source de la vie.
On ne sera donc pas étonné que le récit d'un intellectuel de la stature de Fernand Dumont soit entremêlé, voire souvent occulté par de telles préoccupations essayistiques. Et d'autant plus que la conjonction d'une pudeur sans doute native et d'une volonté expresse de construire un récit qui porte réponse à "quelque ancienne et lancinante préoccupation" (p. 11), le porte à éviter les épanchements. "J'accorderai plus d'attention aux idées qu'aux émotions du moi" (p. 12), annonce-t-il d'emblée. Et ailleurs : "En commençant ce récit, j'avais résolu d'être très discret sur ma vie privée" (p. 201). Ou encore: "À partir de ma jeunesse, j'aurai fait mémoire d'un itinéraire beaucoup mieux par la poésie que par ces réminiscences que je suis en train d'écrire. On peut tout dire à l'abri du poème." ( $p$. 246) Ce qui n'avance guère les amateurs d'une approche rousseauiste de l'autobiographie. Car si l'auteur peut "tout dire" dans le poème, on aura constaté que celui-ci demeure "un abri", c'est-à-dire un écran, un blindage derrière lequel le poète s'abrite et se dérobe. Ces Mémoires sont donc autre chose qu'une confidence, encore qu'elles soient aussi cela pour qui sait lire.

Fernand Dumont s'explique làdessus dans un très beau prologue, page d'anthologie, sous le titre "Pourquoi se souvenir?". Le verbe est ici une synecdoque et désigne, non pas l'acte mémoriel lui-même, largement inconscient, mais plutôt l'action réfléchie de rédiger ces souvenirs :

Les hommes politiques ou les hommes de guerre écrivent des 
mémoires pour commenter des événements qui ont concerné beaucoup de monde. Même leur vie privée a eu quelque rapport avec leurs activités publiques. À l'encontre, l'histoire d'un travailleur de la pensée se confond pour l'essentiel avec celle d'une solitude dont ses livres sont les seuls témoins importants. J'étais donc occupé à l'un de ces ouvrages où on dissimule sa présence quand une terrible maladie s'est abattue de nouveau sur moi. Le courage de l'objectivité m'a fait subitement défaut. Je ne pouvais m'abandonner sans défense à la déréliction; je me raccrochai au seul moyen de diversion que je connaissais, l'écriture. Mais sur quoi écrire quand se dérobe cet appétit du monde qui est aussi oubli de soimême? Je me tournai vers le passé. (p. 11)

On reconnaît dans cet incipit quelques-unes des lois du discours préfaciel (justification initiale, humilité de rigueur, circonstances propices à la remémoration), mais aussi, par un renversement paradoxal, une récupération du vocabulaire guerrier pour désigner une démarche intellectuelle. L'auteur n'est pas un homme de guerre, nous dit-il, et pourtant une thématique militaire s'instaure dès le départ: "on dissimule sa présence", "s'est abattue", "courage"; "m'abandonner sans défense", "moyen de diversion", "se dérobe". Le ton est donné. Cette vie consacrée aux idées et aux théories intellectuelles, à une réflexion épistémologique sur les sciences et sur la pertinence des sciences humaines, nourrie d'une préoccupation constante pour la plus spéculative de toutes celles-ci - la théologie puisqu'elle porte sur une trans- cendance indicible, cette vie qui s'enfonce toujours plus avant sur les "sentiers de l'abstraction" (p. 12), est placée tout entière sous le signe d'une forme particulière de violence, celle d'un "déchirement de l'identité " (p. 11), véritable "tragédie", celle de l'émigration.

Ainsi, le titre de l'œuvre traverse celle-ci comme un fil d'Ariane, multiplie les épiphanies, nouant solidement les parties entre elles, donnant une extrême cohérence au propos. Fernand Dumont est un fils d'ouvrier, son père ayant passé sa vie dans les murs de la Dominion Textiles à Montmorency et ayant logé sa famille dans les "blocs" résidentiels de l'entreprise où l'auteur a vécu son enfance et sa jeunesse; cette famille ouvrière était encore très près de ses racines paysannes dans le comté de Charlevoix. Faire des études, c'était forcément rompre avec cette culture d'origine. Accéder à la culture savante, c'est quitter la culture première. Cette scission est vécue comme une rupture à la fois douloureuse et bénéfique, une rupture souhaitée et subie, un exil inévitable dont on voit les bienfaits mais dont on ne guérit pas. La thématique de l'émigration et de l'exil est ce qui donne son unité à cette œuvre à laquelle siérait mieux, il me semble, et pour cette raison, le soustitre d' autobiographie " plutôt que celui de "mémoires", genre plus éclaté. Ce livre, même inachevé, est très construit.

C'est pourquoi, même si l'œuvre est divisée en huit chapitres bien circonscrits, j'y verrais volontiers deux parties: les trois premiers chapitres consacrés à l'enfance et à la jeunesse, les années préparatoires; 
les cinq suivants consacrés à ce qu'on pourrait appeler la vie publique. Le quatrième chapitre commence en 1955, avec le retour après deux années d'études à Paris. Fernand Dumont a vingt-huit ans, il commence sa vie de professeur à l'Université Laval et sa carrière d'intellectuel. Ces chapitres constituent un témoignage capital sur la vie de jeune professeur au moment où les universités québécoises étaient des institutions cléricales en pleine évolution; on y trouve aussi des pages essentielles sur la constitution des sciences sociales au Québec, sur l'Université Laval en particulier comme milieu intellectuel, sur la communauté exceptionnelle de chercheurs prolifiques qui s'y est créée, sur les années de bouillonnement intense qui ont vu le Québec dans son ensemble passer d'une société catholique relativement homogène à une modernité problématique. Fernand Dumont est un témoin privilégié de ces années. Non seulement parce qu'il fut un compagnon de route de divers mouvements, en particulier ceux d'action catholique ou d'engagement nationaliste, mais surtout parce qu'il a su en devenir un commentateur extrêmement perspicace, se dotant progressivement des outils conceptuels et théoriques qui lui permettraient de comprendre les-événements et de rendre compte de ce qu'il vivait avec ses contemporains.

La volonté de comprendre, de saisir dans une totalité interprétative adéquate traverse ces chapitres, leur confère une tension féconde et une grande intelligence. Fernand Dumont explique dans quel contexte précis s'est faite la gestation de ses livres, à quelles questions intimes et collectives ils essayaient de porter réponse. Il les commente, les résume, les confronte à d'autres. Ainsi, ces chapitres constituent sans doute la meilleure introduction qu'on puisse trouver à son cuvre; du même coup, tant ses intérêts étaient totalisants et sa visée de nature épistémologique, ils sont un excellent survol de l'ensemble des sciences sociales dans leurs ambitions, leurs interrogations, leurs limites, et donc leur dépassement éventuel dans la philosophie et, pourquoi pas, la théologie.

Malgré l'intérêt indéniable de ces chapitres, toutefois, j'avoue candidement qu'ils me semblent pâlir quelque peu à la lumière des premiers. Ceux-ci, moins scolaires, moins énonciatifs, plus personnels donc, sont parcourus d'une émotion contenue mais palpable et sont le fait d'un grand écrivain. Le premier raconte "le pays natal", c'est-à-dire l'enfance dans le giron d'une famille modeste mais riche de chaleur humaine et de valeurs authentiques. Le second décrit "l'exil" progressif occasionné par la lecture fervente, les études secondaires et le difficile passage au cours classique. Le troisième, "une jeunesse au mitan du siècle", raconte les études universitaires, l'éclósion de l'amour, la découverte de la France lors d'un séjour d'études de deux ans. Chacune des étapes apporte sa cohorte de personnages attachants et pittoresques, ses adjuvants rappelés avec affection et gratitude, ses défis particuliers. Le fils reconnaissant, l'élève attentif et déjà marginalisé par ses lectures boulimiques, l'étudiant faisant, émerveillé, de grandes découvertes 
passent successivement sous nos yeux, mais chaque fois le regard est aussi celui du sociologue, de l'historien des mours, du croyant fervent mais angoissé qui ordonnent la mémoire, lui donnent sens et direction.

C'est ainsi, par exemple, que l'enfance est saisie comme une série de cercles concentriques qui se constituent autour du sujet: la famille immédiate et la "tribu " maternelle, d'abord, décrites dans leur identité ouvrière et paysanne; puis le voisinage des résidences corporatives: "différent de la famille, de la tribu, le voisinage était donc très présent lui aussi. Indiscrétions et initiations précoces, certes; mais aussi solidarités, entraide, dont les milieux populaires sont plus capables que d'autres, je crois." (p. 25) ; finalement, troisième cercle, la paroisse avec ses rites, son espace, son clivage socio-économique, son autarcie et son isolement, "à l'écart du vaste monde" (p. 34). "L'exil" des livres, ce "pays nouveau "où son destin le conduit, se constitue aussi en fonction d'un triple espace, intellectuel celui-là: les découvertes décisives de Gaston Bachelard, Marcel Blondel, Emmanuel Mounier. Bachelard lui apprend la liberté de l'esprit et le décloisonnement des connaissances, la ferveur pédagogique qui rend vivant ce qui dort dans les livres; Blondel lui propose une philosophie de l'action et de la responsabilité sans esprit de système; Mounier l'engage à se vouer aux tâches de son temps, à construire une utopie de la culture idéale.

Si j'insiste sur les différents espaces, espaces physiques, espaces intellectuels, c'est sans doute parce que les métaphores spatiales sont omniprésentes dans ce livre: voici une autobiographie où l'espace est plus important que le temps. Cercles de la famille, du voisinage et de la paroisse, enfance qui est un pays, écoles qui sont des "maisons" (p. 57), livres et œuvres comme "pays nouveaux " à découvrir, passerelles à assurer entre les différents lieux: toujours l'espace comme culture, car les clivages spatiaux (les quartiers de Paris, par exemple), sont aussi des clivages culturels, autrement difficiles à franchir que les premiers. S'éduquer, c'est lire ces espaces et essayer de les comprendre, c'est conquérir un nouvel espace sans perdre la mémoire du premier.

Ces chapitres, en somme, décrivent la lente éclosion d'une vocation qui se cherche à tâtons, mais qui trouve néanmoins sur sa route des complicités, des encouragements, des amitiés remarquables. Par ce biais, ces Mémoires renouvellent avec un modèle augustinien de l'autobiographie où le sujet retrace les diverses étapes de la vocation qui lui échoit et dont il doit découvrir les signes sous peine de rater son destin. La vocation, dans ce cas, est intellectuelle, certes. Mais elle est aussi, en conformité avec le modèle, religieuse et spirituelle.
La solitude de l'enfance, un cheminement chaotique à travers les labyrinthes scolaires où je ne pouvais jamais me fier à des routes toutes tracées, des habitudes d'au- todidacte livré à ses impulsions m'avaient conduit à chercher dans les livres et dans l'écriture un palliatif à quelque blessure impossible à guérir. (p. 64)

Blessure qui est celle de l'exil, certes, mais aussi d'une foi religieuse inquiète. Ce n'est pas le moindre 
intérêt de ces Mémoires que de faire une bonne place à la réflexion religieuse et à la quête spirituelle. La chose est trop rare de nos jours pour ne pas être signalée.

Une vie singulière, vécue profondément, fait éclater l'individualité et rejoint l'universel. Toute bonne autobiographie devient un témoignage et un testament qui, audelà du parcours individuel, font entrevoir l'histoire collective. Et davantage. Car, au-dessus de l'histoire, planent d'autres mystères, insondables ceux-là ou du moins fort négligés par les chercheurs. Comment comprendre, par exemple, que le fils d'un ouvrier d'une grande dextérité manuelle, le petit-fils de paysans analphabètes et ratoureux, soit accaparé par des tâches spéculatives et le souci d'une théorisation des sciences sociales, voire la recherche épistémologique. Il y a là un saut existentiel (je ne dis pas qualitatif, qui laisserait supposer une quelconque supériorité de l'intellectuel) qui laisse songeur. Dans sa belle étude de l'œuvre de Brice Parrain, Sartre signalait la chose:

J'ai connu, à l'École Normale, beaucoup de ces fils de paysans que leur intelligence exceptionnelle avait arrachés à la terre. Ils avaient d'énormes silences terriens dont ils sortaient tout à coup pour disserter sur les sujets les plus abstraits... 5

On ne saurait confondre Fernand Dumont avec les Normaliens compa- gnons de Sartre, dont la virtuosité intellectuelle tenait quelque peu de la pédanterie et qui choisissaient de préférence le silence, dans lequel ils retombaient volontiers. Fernand Dumont, au contraire, a choisi, dès le départ, "la parole et l'écriture" (c'est le titre du chapitre IV, le premier de la vie publique), auxquelles il a été d'une remarquable fidélité. Mais le même phénomène d'une parole de l'abstraction théorique, qui se déploie sur fond de silence séculaire, se remarque dans les deux cas. Tout était déjà là, en somme, dans ce peuple dont les intellectuels, ses fils, se détournent trop aisément et envers qui ils ne reconnaissent pas facilement leur dette. Raison de plus, sans doute, pour répondre à l'invitation de Fernand Dumont, exprimé dans ce dernier livre comme dans les études savantes qui l'ont précédé: il faut retrouver notre mémoire. Impossible d'inventer l'avenir en se coupant de sa source vive.

1. Fernand Dúmont, Récit d'une émigration. Mémoires, Montréal, Boréal, 1997, 263 p.

2. Sans compter tous les autres, esprits pratiques, hostiles aux spéculations, qui voyant que Voix et Images a adopté depuis longtemps un classement traditionnellement générique dans ses chroniques (essai, roman, poésie, théâtre), pourraient considérer que par défaut, c'est forcément dans la chronique de l'essai qu'il faut parler de ce livre.

3. Et je conviens volontiers que cette chronique, qui fait aussi souvent écho aux études et aux travaux savants qu'aux véritables essais, n'échappe pas à ce reproche.

4. Hugo Friedrich, Montaigne, Paris, Gallimard, 1968, p. 356.

5. Jean-Paul Sartre, Situations, I, Paris, Gallimard, 1947, p. 181 